\title{
Histomorphological profile of uterine corpus malignancies: a single unit experience
}

\author{
T.S. Waravita and R. M. P. Rathnayake \\ Department of Pathology, Teaching Hospital Kandy, Sri Lanka
}

DOI: http://doi.org/10.4038/jdp.v11i2.7711

\section{Introduction}

Primary uterine malignancy (PUM) is a common malignancy amongst females worldwide. According to the National Cancer Incidence data (2007), the age- specific incidence rate of uterine corpus malignancy is 2.7 per 100000 population in Sri Lanka. The aim of this study was to describe the frequency, clinicopathological and prognostic parameters of PUM.

\section{Methodology}

Hysterectomy specimens (49) and biopsies (05) of PUM received over two years were retrieved to collect demographic and morphological data. The tumours were categorized as epithelial, mesenchymal or mixed. Histological subtypes of epithelial tumours were classified as type 1 (T1) or type 2 (T2). Prognostic markers including grade (1$3)$, stage (FIGO I-IV) and vascular invasion were recorded. The concomitant pathological changes such as endometrial hyperplasia and intra epithelial neoplasia were assessed.

\section{Results}

Fifty four PUM were included. The mean age at presentation was 59 years. There were 45 (83.3\%) endometrial carcinomas (EC), 5(9.3\%) uterine sarcomas, 2(3.7\%) carcinosarcomas (CS) and 2(3.7\%) choriocarcinomas. Of the EC, $86.6 \%(39 / 45)$ were T1 and $11.1 \%(6 / 45)$ were T2. The majority of T1 EC was endometrioid adenocarcinoma (37/45), while 5/45 T2 tumours were serous papillary adenocarcinomas. Ten cases of T1 EC had associated simple (6/10) and complex (4/10) endometrial hyperplasia and 2 cases of T2 EC had intraepithelial carcinoma. Of the endometrioid carcinomas, $13.5 \%$ (5/37), $64.8 \%(24 / 37)$ and $21.6 \%$ (8/37) were grade 1 , 2 and 3 respectively. $3 / 5$ cases of uterine sarcomas were low grade. Of all resection tumours, 63.2\% (31/49), 22.4\% (11/49) and $14.2 \%$ (7/49)) were FIGO stage I, II and III respectively. Of the resected tumours $51.02 \%(25 / 49)$ had more than $50 \%$ of myometrial invasion and $46.93 \%$ (23/49) had vascular invasion.

\section{Conclusion}

T1 endometrial carcinoma was the commonest primary uterine malignancy. Overall, although the patients present at an early stage, they had unfavourable prognostic factors such as deep myometrial invasion, vascular invasion and high histological grade. 